\title{
Variety and Complexity of Surgical Exposure, Operative Autonomy, and Program Reputation Are Important Factors for Orthopaedic Sports Medicine Fellowship Applicants
}

\author{
Francis J. Oser, M.D., Bailey M. Grimsley, B.S., Aubrey J. Swinford, B.S., \\ Symone M. Brown, M.P.H., and Mary K. Mulcahey, M.D.
}

Purpose: To identify the factors considered most important by sports medicine fellowship applicants when deciding where to apply and ultimately interview. Methods: An anonymous, electronic survey was distributed in 2018 via e-mail to orthopaedic surgery residents who applied to a specific orthopaedic sports medicine fellowship program in 2015 and 2016. The survey included questions regarding the number of fellowships applications per respondent and the number of interviews they were offered and accepted. Questions regarding the application process were included. Participants also were asked to rank 9 criteria from most to least important ( 1 being most important; 9 being least important) when deciding where to apply or accept interviews. Each criterion's score reflects its average ranking among respondents. Results: Among the 99 orthopaedic surgery residents applying to this fellowship program, $42(42.4 \%)$ completed the survey. The factors considered most influential included variety and complexity of surgical exposure (2.16), autonomy (3.72), and reputation of faculty members (4.05). The factors considered least important were program size (7.21), job placement of past fellows (7.07), and geographic location (5.68). Conclusions: Variety and complexity of surgical exposure, operative autonomy, and program reputation were valued as the most important factors for orthopaedic surgery residents applying to sports medicine fellowship programs. Clinical Relevance: The information obtained in this study may provide sports medicine fellowship programs and orthopaedic residents with a better understanding of factors that are considered to be important by sports medicine fellowship applicants. This will improve training for future sports medicine specialists, thus improving the care that they provide to their patients.

$\mathbf{I}$ n recent years, orthopaedic sports medicine fellowships consistently have been among the most popular, with more applicants than any other orthopaedic

From Tulane University School of Medicine (F.J.O., B.M.G., A.J.S.); and Department of Orthopaedic Surgery (S.M.B., M.K.M.), Tulane University School of Medicine, New Orleans, Louisiana, U.S.A.

The authors report the following potential conflicts of interest or sources of funding: M.K.M. reports fees from American Academy of Orthopaedic Surgeons, Translational Journal of the ACSM, American Orthopaedic Society for Sports Medicine, Arthrex, Arthroscopy Association of North America, Ortho Info, Ruth Jackson Orthopaedic Society, and The Forum, outside the submitted work. Full ICMJE author disclosure forms are available for this article online, as supplementary material.

Received June 29, 2020; accepted February 10, 2021.

Address correspondence to Mary K. Mulcahey, M.D., 1430 Tulane Ave., \#8632, New Orleans, LA 70118. E-mail: mmulcahey@tulane.edu

(C) 2021 THE AUTHORS. Published by Elsevier Inc. on behalf of the Arthroscopy Association of North America. This is an open access article under the CC BY-NC-ND license (http://creativecommons.org/licenses/by-nc-nd/4.0/). 2666-061X/20992

https://doi.org/10.1016/j.asmr.2021.02.003 subspecialty. ${ }^{1,2}$ Recent studies have demonstrated that roughly $90 \%$ of orthopaedic surgery residents plan to pursue additional training after graduation for various reasons, including surgical skill development, employment opportunities/marketability, and perceived impact of fellowship training on clinical decision-making. ${ }^{3-5}$ Despite the high number of orthopaedic sports medicine fellowship applicants, there is a paucity of information regarding the factors that applicants deem most important when applying. In 2015, Niesen et al. ${ }^{6}$ quantified the factors considered most and least important by residents applying to orthopaedic fellowships in all 9 subspecialties. The factors considered most important were operative experience, autonomy, and fellowship staff members. Research opportunities and salary were deemed least important by applicants. ${ }^{6}$ A recent study conducted by Baweja et al. ${ }^{7}$ identified the most important factors taken into consideration by fellowship program directors (PDs) when ranking sports medicine fellowship applicants. PDs indicated 

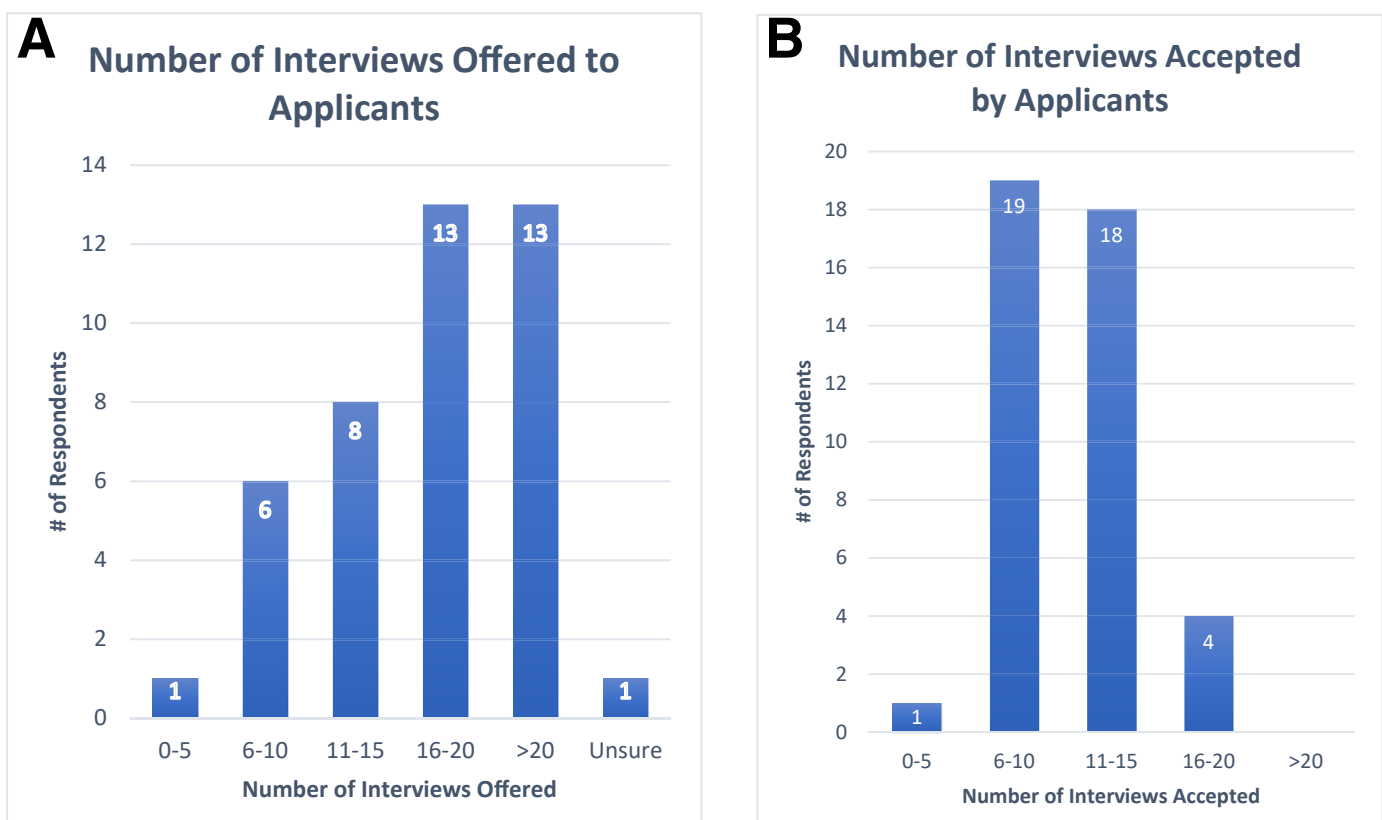

Fig 1. (A) Number of interviews that were offered to applicants applying for a sports medicine fellowship. (B) Number of interviews accepted by applicants applying for a sports medicine fellowship.

that the fellowship interview was the most important factor in ranking applicants, whereas volunteer experience and geographic ties were viewed as the least important criteria when considering a candidate. ${ }^{7}$ Although these studies give insight into the fellowship application process from the perspective of orthopaedic fellowship applicants and sports fellowship PDs, no study has specifically evaluated which program characteristics sports medicine fellowship applicants consider to be most important. The purpose of this study was to identify the factors considered most important by sports medicine fellowship applicants when deciding where to apply and ultimately interview. We hypothesized that the most important factors taken into consideration by orthopaedic sports medicine fellowship applicants was the variety and complexity of surgical exposure.

\section{Methods}

An anonymous, electronic survey consisting of 9 multiple-choice questions was distributed via email to 99 orthopaedic surgeons/residents who applied to the sports medicine fellowship program associated with our institution in 2015 and 2016. The survey was created and distributed using Survey Monkey (SurveyMonkey Inc., San Mateo, CA) in January 2018. The applicants' email addresses were obtained from the fellowship coordinator. Questions included demographic information, ranking a list of 9 criteria from most to least important when deciding where to apply and accept interviews, and additional questions regarding the fellowship interview process. A reminder email was sent 2 and 4 weeks later to encourage more participation. Descriptive statistics were used to evaluate responses.

\section{Results}

Survey responses were obtained from 42 of the 99 $(42.4 \%)$ orthopaedic surgeons/residents who applied to the sports medicine fellowship program associated with our institution in 2015 or 2016. Thirty-seven of 42 $(88.1 \%)$ respondents were male, and 5 were female ( $11.9 \%)$. Thirty-seven of 42 respondents $(88.1 \%)$ have been in practice for less than 1 year $(88.1 \%)$. Of the 42 respondents, $28(66.6 \%)$ applied to more than 20 sports fellowship programs and $26(61.9 \%)$ were offered at least 16 interviews (Fig 1). Thirty-seven of the respondents $(88.1 \%)$ accepted between 6 and 15 interviews from sports medicine fellowship programs (Fig 2 ). Thirty-eight of our respondents $(90.5 \%)$ declined interview offers due to conflicting dates with another interview. Eighteen participants $(42.9 \%)$ turned down interviews due to the inability to get time off from work, and $17(40.5 \%)$ reported declining interview opportunities due to the lack of financial resources (Fig $3)$. The amount of money spent by sports medicine fellowship applicants on the interview process varied considerably from less than $\$ 1000$ to greater than $\$ 10,000$ due to the accumulation of application fees, travel, lodging, and food. Fifteen respondents (35.7\%) spent between $\$ 4001$ and $\$ 6000$ on interviews (Fig 4). In addition to the high price of interviews, more than one-half the participants $(23,54.8 \%)$ missed between 8 


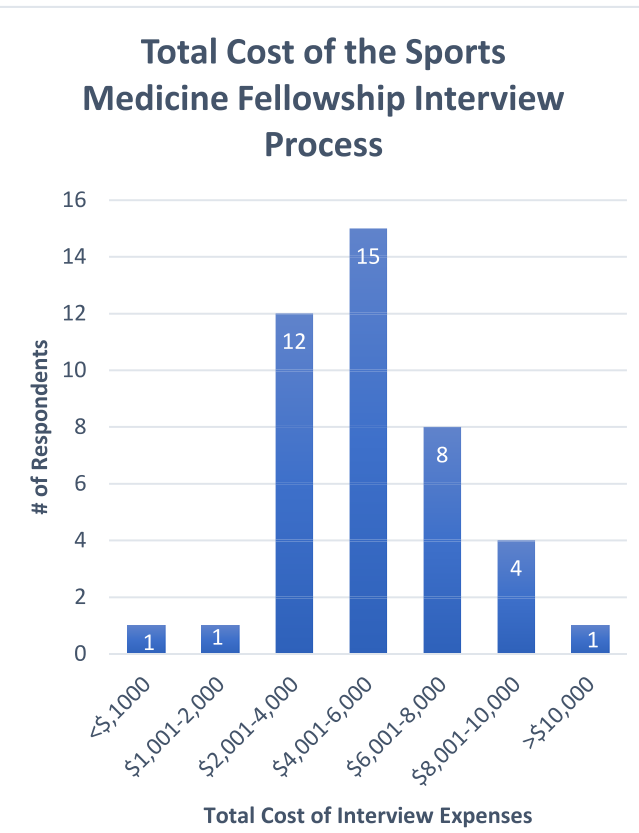

Fig 2. Total amount that applicants spent on the sports medicine fellowship interview process.

and 12 days of residency work due to fellowship interviews.

Participants were asked to rank 9 criteria (listed in order of importance) for selecting a sports medicine fellowship program, with 1 being most important and 9 being least important (Table 1). The most important factors included variety and complexity of surgical exposure (average rank of 2.16), autonomy (3.72), and reputation of faculty (4.05). The least important factors included geographic location (average rank of 5.68), job placement of past fellows (7.07), and program size (i.e., number of fellows per class; 7.21).

\section{Discussion}

The most important factors that applicants consider when applying for an orthopaedic sports medicine fellowship include the variety and complexity of surgical exposure, operative autonomy, and reputation of faculty. These results are similar to those previously reported for orthopaedic surgery residents pursuing fellowship training in a variety of subspecialties. ${ }^{6}$ Program size, job placement of past fellows, and geographic location were factors considered least important by sports medicine fellowship applicants. This study also demonstrated that orthopaedic sports medicine fellowship applicants often are offered interviews from 12 to 20 programs, depending on the number of programs to which the resident applied.

Operative autonomy was a highly influential factor for sports medicine fellowship applicants. The emphasis on operating independently may, in part, be a result of lack of autonomy during residency. The results from our study are similar to findings reported by Niesen et al in 2015. ${ }^{6}$ The authors evaluated postgraduate year (PGY) - 4 and PGY-5 orthopaedic surgery residents in the United States using a 19-question survey distributed through orthopaedic surgery program coordinators. All senior residents were eligible to participate, including those not pursuing fellowship training, with 78 PGY-4 $(52 \%)$ and 71 PGY-5 (48\%) completing the survey out of an estimated total of 1277 residents $(11.6 \%$ response rate). Residents were asked to rank 10 listed criteria in order of importance for selecting a fellowship program and establishing a final rank list. The most important factors were considered to be surgical experience and autonomy (median rank, 2), followed by staff members at the fellowship (median rank, 3). ${ }^{6}$

George et al. $^{8}$ analyzed 14 general surgery programs using the System for Improving and Measuring Procedural Learning, with the goal of establishing levels of competency in their residents as they neared the end of their training. This study had attendings in these programs characterize residents after having observed residents completing procedures throughout the duration of their training. Through this, the authors found that $20 \%$ to $22.6 \%$ of residents were not deemed competent or did not reach meaningful autonomy in their final 6 months of residency. Kelz et al. ${ }^{9}$ studied 30-day outcomes of patients operated on by newly graduated general surgery attendings compared to experienced surgeons. The authors found a significant increase in adverse outcomes including time under anesthesia, length of stay in the hospital, and overall costs for those patients operated on by surgeons who recently graduated from 5 -year general surgery residency programs. These findings support the idea that

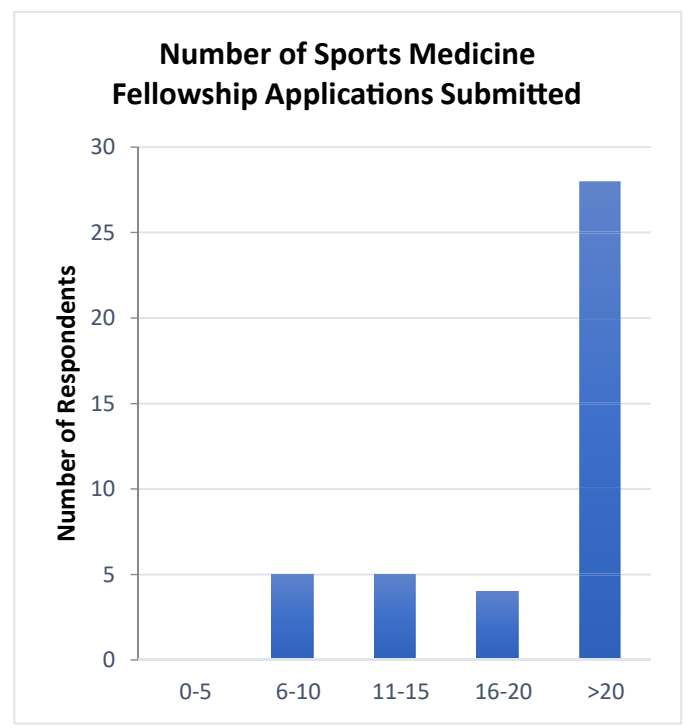

Fig 3. Total number of orthopaedic sports medicine fellowship applications submitted. 


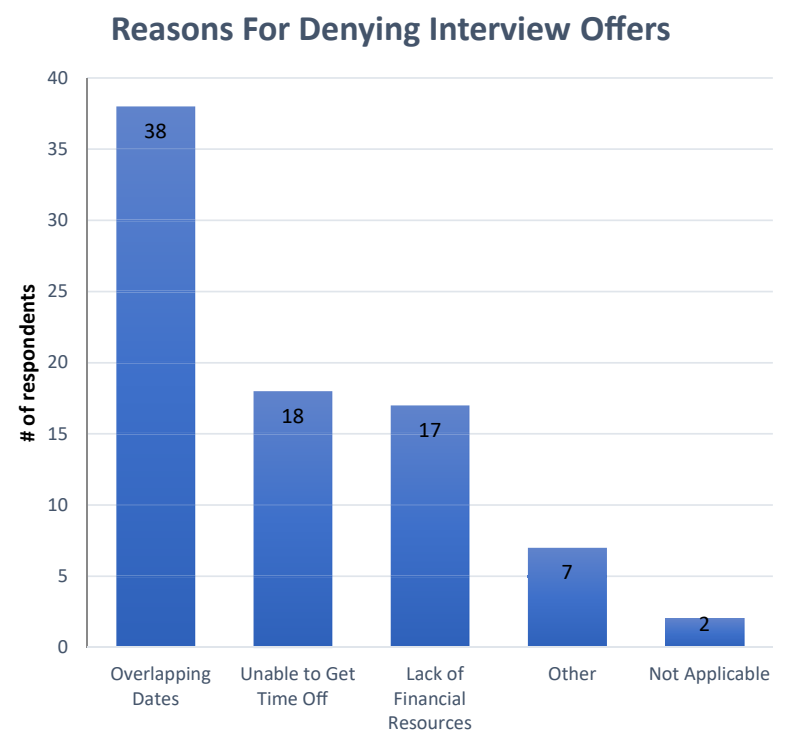

Fig 4. Reasons orthopaedic sports medicine fellowship applicants turned down fellowship interview offers.

residents applying for orthopaedic sports medicine fellowships will value a program that offers more independence in performing surgery to improve their technical skills, in turn, improving outcomes for their patients. However, multiple barriers exist that decrease trainee autonomy and operative experience, including differing views of autonomy between attending physicians and fellows, financial pressures, and duty hour regulations. ${ }^{10}$ In addition, the limit of an 80-hour work week set in 2003 by the Accreditation Council for Graduate Medical Education has led to a significant variability in operative exposure during residency. This suggests a growing disparity in experience among graduating residents and. consequently, a significant difference in the surgical skills and proficiency of these residents. ${ }^{11}$

The prestige of an orthopaedic surgery fellowship program, as well as the reputation of the staff and faculty members, are also considered by applicants when determining which programs to apply to, interview at, and rank. Our results indicate that sports medicine fellowship applicants value the reputation of the faculty over the prestige of the program, which is similar to the findings from Niesen et al. ${ }^{6}$ In their study, prestige of a program was the third highest ranked criteria by orthopaedic surgery fellowship applicants of all subspecialties in constructing their final program ranking (median rank of 4). In addition, applicants from more prestigious residency programs tend to accept interviews to highly reputable fellowships, suggesting that residents value the reputation of the program over many other factors that were widely varied, including location, research opportunities, and operative time. ${ }^{12,13}$ The reputation of the program may be beneficial to obtaining a desirable position after fellowship; however, our study indicated that job placement post-fellowship was rated less important in ranking programs. Prospective fellows may also be drawn to the networking and professional mentorship that usually accompany highly renowned programs.

Of the 42 respondents in our study, $21 \quad(50 \%)$ considered the variety and complexity of surgical exposure offered by sports medicine programs as the most important factor in choosing and ranking fellowship programs. To date, this particular criterion is not well documented in previous studies. However, "variety and complexity of surgical cases" is most similar to the "volume of cases" expected from a fellowship program, and this latter factor has been used in other studies for ranking fellowship programs. Niesen et al. ${ }^{6}$ demonstrated that the number of cases was the fourth most important factor in ranking orthopaedic fellowship programs with a median ranking of 4 . We believe our criterion established a higher ranking in the view of fellowship applicants because it implies not necessarily a larger volume, but rather more exposure to cases besides only the most common sports medicine procedures.

\section{Limitations}

There are several limitations to this study. This is a survey-based study and hence, it is subject to all limitations inherent to this type of study design. The survey was sent to a group of 99 residents who applied to 1 fellowship program over a 2 -year period, and their response may not reflect the opinions or experiences of all residents who applied for sports fellowships during the same time period. Also, only 42 of the 99 residents $(42.4 \%)$ responded to the survey, and 4 participants did not fully answer the first survey question, which may have resulted in a skewed breakdown of the answers for that question. Information used by applicants when

Table 1. Average Rankings* of the Factors Considered by Applicants When Applying to and Interviewing for Orthopaedic Sports Medicine Fellowship

\begin{tabular}{lc}
$\begin{array}{l}\text { Factors Considered by Sports Medicine Fellowship } \\
\text { Applicants }\end{array}$ & Avg. Ranking \\
\hline Variety and complexity of surgical exposure & 2.16 \\
Autonomy & 3.72 \\
Reputation of staff/faculty members & 4.05 \\
Satisfaction of past fellows & 4.37 \\
Program prestige & 5.26 \\
Involvement with collegiate/professional & 5.62 \\
sports team during fellowship & \\
Geographic location & 5.68 \\
Job placement of past fellows & 7.07 \\
Program size & 7.21
\end{tabular}

*Average ranking is calculated as a mean of the applicants' responses (" 1 " indicates the most important criteria; "9" signifies the least important criteria). 
deciding where to apply and interview was likely from second-hand resources, such as reddit, blog posts from previous applicants, and co-residents who may have previously visited the same program. Often, this information is not directly from the fellowship programs; therefore, it is unlikely that all applicants had access to the same information throughout the fellowship application process. Lastly, there is the potential for recall bias due to the multi-year gap between when the residents applied for fellowship and when the survey was distributed to them.

\section{Conclusions}

Variety and complexity of surgical exposure, operative autonomy, and program reputation were valued as the most important factors for orthopaedic surgery residents applying to sports medicine fellowship programs.

\section{References}

1. Oladeji LO, Pehler SF, Raley JA, Khoury JG, Ponce BA. Is the orthopedic fellowship interview process broken? A survey of program directors and residents. Am J Orthop 2015;44:444-453.

2. Yin B, Gandhi J, Limpisvasti O, Mohr K, ElAttrache NS. Impact of fellowship training on clinical practice of orthopaedic sports medicine. J Bone Joint Surg Am 2015;97:e27.

3. Almansoori KA, Clark M. Increasing trends in orthopedic fellowships are not due to inadequate residency training. Educ Res Int 2015;2015:1-9.

4. Haislup BD, Kraeutler MJ, Baweja R, McCarty EC, Mulcahey MK. Orthopaedic sports medicine fellowship interviews: Structure and organization of the interview day. Orthop J Sports Med 2017;5:2325967117741276.
5. Horst PK, Choo K, Bharucha N, Vail TP. Graduates of orthopaedic residency training are increasingly subspecialized: A review of the American Board of Orthopaedic Surgery part II database. J Bone Joint Surg Am 2015;97:869-875.

6. Niesen MC, Wong J, Ebramzadeh E, et al. Orthopedic surgery fellowships: The effects of interviewing and how residents establish a rank list. Orthopedics 2015;38:175-179.

7. Baweja R, Kraeutler MJ, Mulcahey MK, McCarty EC. Determining the most important factors involved in ranking orthopaedic sports medicine fellowship applicants. Ortho J Sports Med 2017; 5:2325967117736726.

8. George BC, Bohnen JD, Williams RG, et al. Readiness of US general surgery residents for independent practice [published correction appears in Ann Surg 2018;267:e63]. Ann Surg 2017;266:582-594.

9. Kelz RR, Niknam BA, Sellers MM, et al. Duty hour reform and the outcomes of patients treated by new surgeons. Ann Surg 2020;271:599-605.

10. Hashimoto DA, Bynum WE, Lillemoe KD, Sachdeva AK. See more, do more, teach more: Surgical resident autonomy and the transition to independent practice. Acad Med 2016;91:757-760.

11. Quillin RC 3rd, Cortez AR, Pritts TA, Hanseman DJ, Edwards MJ, Davis BR. Operative variability among residents has increased since implementation of the 80-hour workweek. J Am Coll Surg 2016;222:1201-1210.

12. Krueger CA, Helms JR, Bell AJ, Israel H, Cannada LK. How the reputation of orthopaedic residency programs is associated with orthopaedic fellowship match results. J Bone Joint Surg Am 2020;102:e28.

13. Krueger CA, Chisari E, Israel H, Cannada LK. Residency program reputation influences the American Association of Hip and Knee Surgeons match results. J Arthroplasty 2020;35:2676-2681. 